\title{
Care of terminally-ill patients: an opinion survey among critical care healthcare providers in the Middle East
}

\author{
ur Rahman $\mathrm{M}^{1}$, Abuhasna $\mathrm{S}^{1}$, Abu-Zidan $\mathrm{FM}^{2}$ \\ 1. Tawam Hospital in affiliation with Johns Hopkins, Al-Ain, United Arab Emirates \\ 2. Trauma Group, Faculty of Medicine \& Health Sciences, United Arab Emirates University, Al-Ain, United \\ Arab Emirates
}

\begin{abstract}
Background: Modern medicine has allowed physicians to support the dying terminally-ill patient with artificial means. However, a common dilemma faced by physicians in general, and intensivist in particular is when to limit or withdraw aggressive intervention.

Objective: To study the effect of training background and seniority on Do-not to resuscitate (DNR) decisions in the Middle East.

Methods: Anonymous questionnaire sent to members of the Pan Arab Society of Critical Care.

Results: The response rate was $46.2 \%$. Most of the responders were Muslim (86\%) and consultants $(70.9 \%)$. Majority of the responders were trained in western countries. Religion played a major role in $59.3 \%$ for making the DNR decision. DNR was considered equivalent to comfort care by $39.5 \%$. In a futile case scenario, Do Not Escalate Therapy was preferred (54.7\%). The likelihood of a patient, once labeled DNR, being clinically neglected was a concern among $46.5 \%$. Admission of DNR patients to the ICU was acceptable for $47.7 \%$. Almost one-half of the responders (46.5\%) wanted physicians to have the ultimate authority in the DNR decision. Training background was a significant factor affecting the interpretation of the term no code DNR $(\mathrm{P}<0.008)$.

Conclusion: Training background and level of seniority in critical care provider does not impact opinion on most of end of life issues related to care of terminally-ill patients. However, DNR is considered equivalent to comfort care among majority of Middle Eastern trained physicians.

Keywords: Do-not resuscitate, Islam, care of terminally-ill, opinion, training.

African Health Sciences 2013; 13(4): 893 - 898 http://dx.doi.org/10.4314/ahs.v13i4.5
\end{abstract}

\section{Introduction}

Modern medicine has allowed physicians to support the dying terminally-ill patient with artificial means. However, a common dilemma faced by physicians in general, and intensivist in particular is when to limit or withdraw aggressive intervention.

DNR order is a sensitive topic from religious, social and cultural aspects. Understandably, there is lack of agreement on what is the correct way to deal with this ethical and moral issue. Western countries have been grappling with this subject since cardiopulmonary resuscitation (CPR) was introduced in 1960s'. In the United States, DNR order has

\begin{tabular}{|l|}
\hline *Corresponding author: \\
Fikri Abu-Zidan \\
Professor, Head, Trauma Group, Department of \\
Surgery \\
Faculty of Medicine and Health Sciences \\
UAE University \\
PO Box 17666, Al-Ain, United Arab Emirates \\
Tel: (+971) 37137579 \\
Fax: (+9713)7672067 \\
E-mail: fabuzidan@uaeu.ac.ae
\end{tabular}

African Health Sciences Vol 13 Issue 4 December 2013 evolved and medicine has moved from a paternalistic model to one that promotes autonomy and selfdetermination ${ }^{1,2}$. Conversely, in Europe patientphysician relationship is still paternalistic ${ }^{3-5}$.

Demographics of physicians in the Middle East in general, and the Gulf Cooperating Countries (GCC) in particular, are unique. Majority of the physicians are originally from the Middle East or the Indian Sub-continent. However, the training background varies with consultants (attending) who are primarily western trained while the vast majority of junior staff (medical officers or specialists) is generally trained in the Middle East.

Majority of countries in the Middle East in general and hospitals in particular, do not have a defined DNR policy.

In general, physicians' opinion plays as a major role in managing terminally-ill patients. Patient's age, diagnosis, ICU stay and religious factors have been identified as factors that formulate opinion on patient's codes status ${ }^{6}$. In the Middle East, doctors have to consider religious and cultural issues more than economic considerations when taking the 
DNR decision ${ }^{7}$. The strength of DNR order recommendations varies with medicine specialty and years of training and experience ${ }^{8}$. However, to our knowledge, the influence of western versus Middle Eastern training background among critical care providers on the DNR decision has not been looked at. We aimed to study the affect of training background and seniority on the perception of critical care healthcare providers on DNR decisions in the Middle East.

\section{Methods}

A questionnaire was sent via an e mail to all members of The Pan Arab Critical Care Society $(n=186)$ between October 2007 to Jan 2008. We based our questionnaire on a study done in Europe ${ }^{4}$. The questionnaire was modified according to our local needs.

Data were collected regarding socio-demographic characteristics including age, gender, religion (Muslim, non Muslim), country of training, current position (consultant, specialist, medical officer or resident), and specialty.

The questionnaire asked the opinion of health care providers on DNR/ no code and related ethical issues that arise during daily practice. The effect of training background and religion on the health care providers' opinion was studied.

\section{Statistics}

Fisher's Exact test was used to compare categorical data of two independent groups.

$P$ value of less than 0.05 was considered significant. Data were analyzed using PASW statistics 18, SPSS Inc, USA.

\section{Results}

A total of 86 members filled the questionnaire, (Response rate 46\%). $92 \%$ percent of the respondents were males while $95 \%$ percent were physicians. Majority were between the ages of 4050 years. Eighty six percent of the respondents had a Muslim religious back ground. Majority (34\%) of responders were primarily trained in the Middle East followed by North America (29\%) and Europe (17.4\%) (table 1).
Table 1: Background training for responders of Pan Arab Critical Care Society $(n=86)$.

\begin{tabular}{lll}
\hline Country of training & $\begin{array}{l}\text { Number } \\
(\mathbf{n}=\mathbf{8 6})\end{array}$ & $\mathbf{\%}$ \\
\hline Middle Eastern & 29 & 33.7 \\
Canada & 15 & 17.4 \\
Europe & 15 & 17.4 \\
USA & 10 & 11.6 \\
India & 7 & 8.1 \\
Pakistan & 3 & 3.5 \\
Australia & 3 & 3.5 \\
Others & 4 & 4.7 \\
\hline
\end{tabular}

Majority $(70.9 \%)$ were consultants followed by staff physicians. Most of the responders (64\%) were involved in making DNR decisions more often than once a month. For $59.3 \%$ of the responders, religion played a major role in making the DNR decision. While, for $39.5 \%$ DNR Patient was equivalent to comfort care. In terminal cases, do not escalate was favored by majority $(54.7 \%)$, followed by withdrawal $(20.9 \%)$, while continuing maximum therapy including CPR was preferred by $12.8 \%$. Likelihood of the DNR patient being clinically neglected was a major concern (46.5\%). Almost half $(46.5 \%)$ of the responders wanted physicians to have the ultimate authority to over ride the wishes of the family. Majority $(62.2 \%)$ of the hospitals of responders did not have a formal DNR policy. Importance of comfort during dying was priority for $45.3 \%$, and ability to pray while dying was main concern for $52.3 \%$. Admission of DNR patients to ICU was acceptable by $47.7 \%$. Majority agreed on feeding DNR patients (94\%). The best time to discuss end of life issues was prior to patient getting severely ill was favored in $60.5 \%$.

There was no significant effect of training background and seniority on management plan, neglect of patient, right to override opinion of family, the best defining conditions of patient's death or abuse of the code. Nevertheless, place of training had a significant effect on the interpretation of term "DNR/no code" ( $p<0.008$, Fisher's exact test). 31/46 western trained providers defined it as patients should be treated maximally short of CPR and intubation, while $20 / 39$ of the non western trained defined it as patient should have no investigation and made comfortable. 
Table 2: Effect of background training on DNR opinion

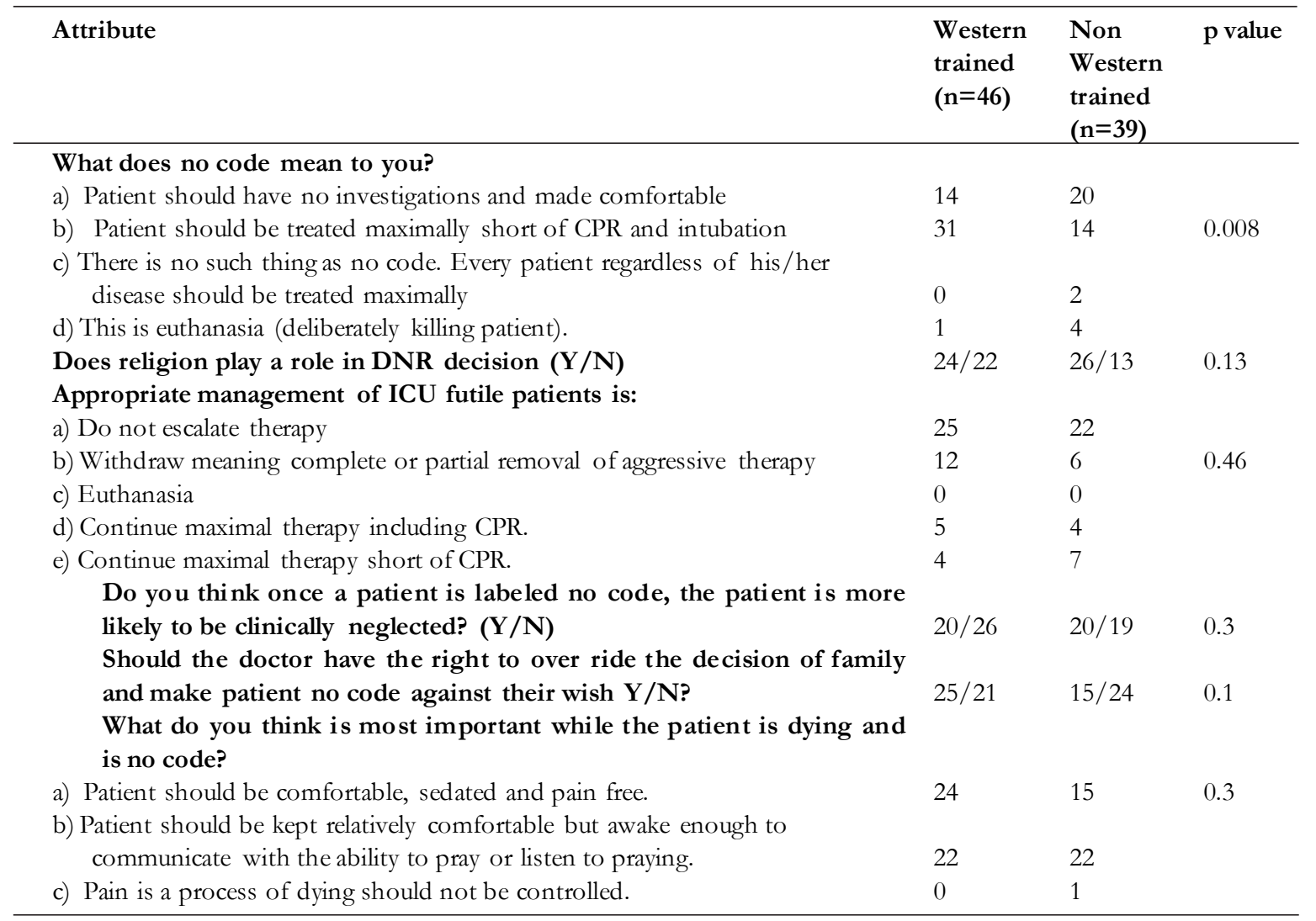

Table 3: Effect of seniority on DNR opinion

\begin{tabular}{|c|c|c|c|}
\hline Attribute & $\begin{array}{l}\text { Consultant } \\
(n=53)\end{array}$ & $\begin{array}{l}\text { Non } \\
\text { consultant } \\
(n=33)\end{array}$ & $\begin{array}{l}\mathrm{p} \\
\text { value }\end{array}$ \\
\hline \multicolumn{4}{|l|}{ What does no code mean to you? } \\
\hline a) Patient should have no investigations and made comfortable & 17 & 17 & \\
\hline b) Patient should be treated maximally short of CPR and intubation & 33 & 13 & 0.35 \\
\hline $\begin{array}{l}\text { c) There is no such thing as no code. Every patient regardless of his/her disease } \\
\text { should be treated maximally }\end{array}$ & 1 & 1 & \\
\hline d) This is euthanasia (deliberately killing patient). & 2 & 2 & \\
\hline Does religion play a role in DNR decision $(\mathrm{Y} / \mathrm{N})$ & $34 / 19$ & $17 / 16$ & 0.09 \\
\hline \multicolumn{4}{|l|}{ Appropriate management of ICU futile patients is: } \\
\hline a) Do not escalate therapy & 30 & 17 & \\
\hline b) Withdraw meaning complete or partial removal of aggressive therapy & 10 & 8 & 0.94 \\
\hline c) Euthanasia & 0 & 0 & \\
\hline d) Continue maximal therapy including CPR. & 6 & 4 & \\
\hline e) Continue maximal therapy short of CPR. & 7 & 4 & \\
\hline $\begin{array}{l}\text { Do you think once a patient is labeled no code, the patient is more likely } \\
\text { to be clinically neglected? (Y/N) }\end{array}$ & $24 / 29$ & $16 / 17$ & 0.47 \\
\hline $\begin{array}{l}\text { Should the doctor have the right to over ride the decision of family and } \\
\text { make patient no code against their wish } \mathrm{Y} / \mathrm{N} \text { ? }\end{array}$ & $27 / 26$ & $13 / 20$ & 0.2 \\
\hline \multicolumn{4}{|l|}{$\begin{array}{l}\text { What do you think is most important while the patient is dying and is no } \\
\text { code? }\end{array}$} \\
\hline a) Patient should be comfortable, sedated and pain free. & 26 & 13 & 0.26 \\
\hline $\begin{array}{l}\text { b) Patient should be kept relatively comfortable but awake enough to } \\
\text { communicate with the ability to pray or listen to praying. }\end{array}$ & 27 & 18 & \\
\hline c) Pain is a process of dying should not be controlled. & 0 & 1 & \\
\hline
\end{tabular}




\section{Discussion}

To our knowledge this is the first study which looks at the impact of western versus Middle Eastern training background and level of seniority among critical care health care providers on end of life issues in terminally-ill patients.

The study demonstrates that end-of life actions are a common occurrence in The Middle East hospitals. However, most of the intensivists work in hospitals where there is no formal DNR policy. This leads to a variety of practices and approaches to handle end of life issue in terminallyill patients.

In our study religion played a significant role in DNR decision by majority of responders (table 2-3). Physicians' training background or level of seniority had no significant impact on the role of religion when deciding DNR issues. It is noted that majority of the responders were Muslims trained in different parts of the world. However, religion continued to play an important role in their daily practice. Like Christianity and Judaism, Islam acknowledges that the death is the inevitable phase of life of human beings. Medical management should not be given if it prolongs the final stage of a terminal illness as opposed to treating a superimposed, life-threatening condition ${ }^{9}$. However, Islam believes that all healing comes from God, so Man has an obligation to search medical care and right to receive appropriate medical treatment ${ }^{10}$. Interestingly, our findings are similar to the previous questionnaire done on predominantly Christian physicians in Europe ${ }^{4,11}$. We did not go into details of religious beliefs such as practicing versus non practicing. However, we believe majority of responders had a religious inclination as religious belief played a decisive role for majority when deciding on limiting therapy in critically-ill patients.

Physicians with Western versus Middle Eastern training background had significantly different opinion on the meaning of DNR/no code (table 2). Middle East trained physicians preferred limitation of therapy and comfort for DNR patient as compared to western trained physicians $(\mathrm{P}<$ 0.008). This may indirectly mean, for physician, comfort is the priority for the dying patient. It also reflects the paternalistic environment that may exist in the Middle Eastern culture. Though again it varies from center to center and country to country ${ }^{12,13}$. We cannot exclude different interpretations among health care providers in understanding the meaning no code, which unlike comfort care does not means "total cessation of active medical management" including blood investigation or medical treatment. Perhaps, western trained physicians have more exposure to medico legal aspects and interpretation of these different medical terms to limit therapy. Conceivably, further awareness and education is needed among Middle Eastern trained physicians to clarify the difference between of DNR/no code and comfort care.

Withholding medical therapy in terminallyill patients is now been widely accepted in around the world on medical, legal, ethical, and moral grounds ${ }^{4}$. Critical care physicians and other health care providers have to base their recommendations on scientific data so as to limit treatment in case of medical futility ${ }^{14}$.

Most of the physicians in our study favored not to escalate but to continue the ongoing management without adding any additional therapy. However, only 13\% accepted withdrawal. Background training or level of seniority did not have any significant impact on the decision to withhold or withdraw therapy (tables 2 and 3). There may be a feeling among physicians of hastening death when therapy is withdrawn, which may go against their religious belief. Euthanasia is totally unacceptable in Middle Eastern culture. The law in Middle Eastern countries does not support the concept of assisted suicide or mercy killing or euthanasia. Any physician who engages in such process would be subjected to legal proceeding on account of murder ${ }^{15}$. Withholding therapy is more acceptable among physicians as doctors in general withhold information about interventions judged too futile to offer. They thus keep greater decision-making control and face weaker obligations to obtain consent from patients or proxies. Withdrawal of care obligates the doctor to include patients (or proxies), even when continued life support is considered fruitless ${ }^{16}$.

There were a significant number of responders who were concerned about clinical neglect of patients once labeled DNR (table 2,3). This again could be due to misinterpretation of the term DNR being equated to comfort care which leads to less intense medical and nursing care for patients labeled no code.

Intensivists practicing in the Middle East agree overwhelmingly on not to stop feeding in terminally-ill patients (table 2 and 3). Patient's well being is highly regarded in the Middle Eastern culture. Food is considered a basic need and right of the patient so the group was almost unanimous to feed and hydrate dying patients. Probably holding feed 
without a good reason was considered deliberate withdrawal of basic need and hence hastening death which is not acceptable.

The patient - physician relationship varies in the United States and Europe. Paternalism remains prevalent in Europe ${ }^{4}$. Medical decisions are based primarily on the opinion of the physicians rather than on the wishes of the patient or their family members. On the contrary, the end of life issue in the United States have evolved and moved from paternalist to be patient-centered. In our survey, physicians were equally split on overriding the wishes of the family on no code decision (table 2 and 3 ). This suggests the present patient -physician relationship in the Middle East is a mix between paternalistic and patient automony. Again, training background and level of training had no significant impact on the DNR decision.

Admitting a dying patient with poor prognosis to ICU is highly controversial and has caused a lot of debate. In our survey background training or level of seniority had no impact on the opinion for admission to ICU of DNR patient. For some clinicians, DNR means do not admit to ICU. However, to our surprise $50 \%$ of responder are willing to accept the DNR patient to ICU. This number is quite similar to other surveys done in Western world ${ }^{5,11}$. DNR does not mean do not treat. Patients who are labeled DNR due to non terminal disease such as early dementia, end stage heart disease or patients with unclear advanced directive are perfect examples of patients who deserve ICU admission for a reversible or treatable condition requiring intensive medical treatment.

Intensive care units in the Middle East are increasingly faced with the issue of admitting and managing terminally-ill patients ${ }^{17}$. There is no clear agreement on when and how to deal with end of life issues. There are no clear guidelines or obvious legal protection for the physicians. Most of the countries do not have clear law, and even in countries where there is DNR policy, it varies from institute to institute ${ }^{15,18}$. In our survey majority of the responder were working in hospitals with no formal DNR policy. However, some hospitals have taken a lead and have implemented a formal DNR policy resulting in clear DNR orders written in majority of dying patients ${ }^{18}$.

To our knowledge this is the first study to compare the opinion of intensivists with western versus Middle Eastern training background and seniority level on end of life care issues in critical terminally-ill patient. Our study had a small sample size. However, we believe that it reflects an appropriate segment of physicians that generally deals with the terminally-ill patient in the ICU. Further studies on end of life issues in Middle Eastern countries are needed so that this region can come to grapple with this controversial but vital topic.

\section{Conclusion}

Intensive care providers from varying training backgrounds and seniority level, in the Middle East, agree on most of the issues on managing terminallyill patients. Limiting therapy is a new concept in the Middle East with no legal definition therefore there may be ambiguity in interpreting the term no code and comfort care among Middle East trained physicians. Majority want to be the primary decision makers while making patient DNR without compromising patient's and family autonomy. Euthanasia is not acceptable culturally and legally. The most acceptable mode of limiting therapy is no escalation while continuing present therapy.

\section{Competing interests}

Authors declare no conflict of interest, i.e. personal, professional or business affiliation relevant to the paper.

This paper has been presented as a poster at the 28th International Symposium on Intensive Care and Emergency Medicine, 18-21 March 2008, Brussels, Belgium and published in abstract form in Critical Care 2008, 12(Suppl 2): P519doi:10.1186/ cc6740

\section{References}

1. Teres D. Trends from the United States with end of life decisions in the intensive care unit. Intensive Care Med 1993; 19:316-322

2. Task Force on Ethics of The Society of Critical Care Medicine: Consensus Statement on the ethics of foregoing life-sustaining treatments in the critically ill. Crit Care Med 1990; 18:1435-1439

3. Richter J, Eisenmann M, Zgonnika E. Doctors' authoritarianism in end of life treatment decisions: a comparison between Russia, Sweden and Germany. J Med Ethics 2001; 27:186-191

4. Vincent JL: Forgoing life support in western European intensive care units: the results of an ethical questionnaire. Crit Care Med 1999; 27:16261633 
5. Ferrand E, Robert R, Ingrand $P$, et al. Withholding and withdrawing of life support in intensive care units in France:a prospective survey. Lancet 2001; 357:9-14.

6. Sprung CL, Cohen SL, Sjokvist P, et al. Endof-Life Practices in European Intensive Care Units The Ethicus Study. JAMA 2003; 290:790797.

7. Da Costa DE, Ghazal H, Al Khusaiby S. Do Not Resuscitate orders and ethical decisions in a neonatal intensive care unit in a Muslim community. Arch Dis Child Fetal Neonatal Ed 2002; 86:F115-F119

8. Kelly WF, Eliasson AH, Stocker DJ, et al. Do specialists differ on do-not-resuscitate decisions? Chest 2002; 121:957-63.

9. Hedayat K, Pirzadeh R. Issues in Islamic biomedical ethics. A primer for the pediatrician. Pediatrics 2001; 108:965

10. Mavani H. A Guide to Islamic Medical Ethics Montreal, QC, Canada: Organization for the Advancement of Islamic Knowledge and Humanitarian Services 1998; 374-436

11. Cardosa T, Fonseca T, Pereira S, et al. Life sustaining treatment decision in Portuguese Intensive care units: A National Survey of intensive care physicians. Crit Care 2003; 7: R167R175
12. Adib S, Hanadeh G. Attitudes of Lebanese public regarding disclosure of serious illness. $J$ Med Ethics 1999; 25:399-403

13. Mobeireek AF, Al Kassimi FA, Al Majid SA, et al. Communication with the seriously ill: Physicians attitudes in Saudi Arabia. JMed Ethics 1996; 22:282-285

14. The Ethics Committee of the Society of Critical Care Medicine. Consensus statement of the Society of Critical Care Medicine's Ethics Committee regarding futile and other possibly inadvisable treatments. Crit Care Med 1997; 25:888-891

15. Takrouri M, Halwani T. An Islamic Medical and Legal Prospective of Do Not Resuscitate Order In Critical Care Medicine. The Internet Journal of Health 2008; 7:1.

16. Gedge E, Giacomini M, Cook D. Withholding and Withdrawing life support in critical care settings: ethical issues concerning consent. J Med Ethics 2007; 33:215-218.

17. Arabi $\mathrm{Y}, \mathrm{Al}$ Shemmeri A. Improving resource utilization in the intensive care units: A challenge for Saudi Hospitals. Saudi Med J 2003; 24: 131137.

18. Rahman M, Arabi Y, Adhami N. et al. Current practice of Do-Not-Resuscitate (DNR) orders in a Saudi Arabian tertiary care center (Abstract). Critical Care 2001; 5: S121 